PROCEEDINGS OF THE

AMERICAN MATHEMATICAL SOCIETY

Volume 126, Number 7, July 1998, Pages 2131-2139

S 0002-9939(98)04314-7

\title{
ENUMERATIONS, COUNTABLE STRUCTURES AND TURING DEGREES
}

\author{
STEPHAN WEHNER
}

(Communicated by Andreas R. Blass)

\begin{abstract}
It is proven that there is a family of sets of natural numbers which has enumerations in every Turing degree except for the recursive degree. This implies that there is a countable structure which has representations in all but the recursive degree. Moreover, it is shown that there is such a structure which has a recursively represented elementary extension.
\end{abstract}

\section{INTRODUCTION}

In the following we are concerned with countable structures in a recursive language. Researchers have investigated how one could measure the intuitive idea of information content of such structures and tried to relate each one of them to a Turing degree [2], [3], [1]. The natural starting point is to look at the collection of representations. Let $\mathfrak{A}$ be a structure. If $\mathfrak{B}$ is an isomorphic structure with universe $\omega$, then $\mathfrak{B}$ is called a representation of $\mathfrak{A}$ (written $\mathfrak{B} \simeq \mathfrak{A}$ ). $D(\mathfrak{B})$, its open diagram, can be regarded as a subset of $\omega$ so that it has a Turing degree, and one can look at the collection of degrees $\{\operatorname{deg}(D(\mathfrak{B})): \mathfrak{B} \simeq \mathfrak{A}\}$. A first guess for capturing the complexity of $\mathfrak{A}$ would be to let its degree be the least element of this collection, especially in the light of the following theorem [2, Theorem 4.1]:

Theorem 1.1 (Knight). Let $\mathfrak{A}$ be a structure in a relational language. Then exactly one of the following holds: (1) For any $\mathbf{d}>\operatorname{deg}(D(\mathfrak{A}))$, there is a representation $\mathfrak{B}$ of $\mathfrak{A}$ such that $\operatorname{deg}(D(\mathfrak{B}))=\mathbf{d}$. (2) There is a finite subset $S$ of the universe of $\mathfrak{A}$ such that all permutations of the universe which fix $S$ are automorphisms of $\mathfrak{A}$.

But this idea fails. For example, Richter [3, Theorem 3.3] shows that for any countable order $\mathfrak{C}$ which has no recursive representation the collection $\{\operatorname{deg}(D(\mathfrak{B}))$ : $\mathfrak{B} \simeq \mathfrak{C}\}$ has no least element. Therefore more involved concepts have been tried to assign degrees to structures [1].

Now for the particular problems addressed in this paper. Steffen Lempp asked (unpublished): Does a structure with representations in all non-recursive degrees have a recursive representation? Julia Knight asked some related questions: With a binary relation $R \subseteq \omega^{2}$ associate a family of subsets of $\omega$ given by $\mathcal{F}_{R}:=\left\{R_{n}\right.$ : $n \in \omega\}$, where $R_{n}:=\{x:(n, x) \in R\}$; say that $R$ is an enumeration of $\mathcal{F}_{R}$. She

Received by the editors September 17, 1996 and, in revised form, January 6, 1997.

1991 Mathematics Subject Classification. Primary 03D45.

Many thanks go to Julia Knight and Carl Jockusch! 
asked (also unpublished): If a family $\mathcal{F}$ has the feature that for every non-recursive set $X, \mathcal{F}$ has an enumeration recursive in $X$, does $\mathcal{F}$ have a recursive enumeration? Similarly, if for every non-recursive set $X, \mathcal{F}$ has an enumeration r.e. in $X$, does $\mathcal{F}$ have an r.e. enumeration?

In the next section we give some positive results on Knight's questions under extra hypotheses. In Section 3 we prove that the answer to Knight's questions is negative, by constructing a single suitable family. This implies that Lempp's question also has a negative answer, as is shown in the last section. The same finding is obtained in [4], but by another approach. We close by discussing the difference.

The notation is quite standard and follows [5]. All sets considered are subsets of $\omega$, the set of natural numbers. We call countable collections of subsets of $\omega$ families. Let $\varphi$ be a Gödel-numbering of the partial recursive functions (of varying arity) and $W_{i}:=\operatorname{Rng}\left(\varphi_{i}\right)$ be an enumeration of the recursively enumerable sets as usual. $W_{i, s}$ is the set of numbers enumerated in $W_{i}$ by stage $s$. We use recursive bijections $\langle\cdot, \cdot\rangle,\langle\cdot, \cdot, \cdot\rangle$ between $\omega$ and $\omega^{2}, \omega$ and $\omega^{3}$, respectively. We also use projections (.). ${ }_{1}$ and (.).2 so that, for example, $(\langle a, b\rangle)_{.1}=a$. Let $\langle x, A\rangle$ be the set $\{\langle x, a\rangle: a \in A\}$. Similarly, $A_{.2}:=\left\{(a)_{.2}: a \in A\right\}$. We let $A+x=\{a+x: a \in A\}$ and $A-x=\{b: b+x \in A\}$.

Fix an effective listing $\Omega$ of recursively enumerable enumerations of all families with r.e. enumerations:

$$
\Omega^{(e)}:=\left\{(i, x):\langle i, x\rangle \in W_{e}\right\},
$$

and write $\Omega_{i}^{(e)}$ for $\left\{x:\langle i, x\rangle \in W_{e}\right\}$, the $i$-th set of the enumeration $\Omega^{(e)}$. Define $\mathcal{C}^{(e)}:=\left\{\Omega_{i}^{(e)}: i \in \omega\right\}$ to be the family enumerated by $\Omega^{(e)}$.

$D: \omega \rightarrow 2^{\omega}$ denotes the canonical enumeration of the family of finite sets; write $D_{n}$ for the $n$-th finite set. Then the binary predicates $x \in D_{n}$ and $x=\left|D_{n}\right|$ are recursive.

\section{Positive Results}

In this section we give conditions on a family which ensure that the implications of Knight's two questions hold. These are given in Theorems 2.3 and 2.4 and are due to Julia Knight. Jockusch gave a proof of Theorem 2.4 which also showed the following: There is a property which families with a recursive (r.e.) enumeration share with families that have, for all non-recursive degrees $\mathbf{d}$, an enumeration recursive (r.e.) in $\mathbf{d}$. These seem to be the only positive statements possible about such families.

What is this property? By a rather straightforward forcing construction it follows that if a set of natural numbers is recursive (r.e.) in all non-recursive degrees, then it is recursive (r.e.). Therefore, the members of a family are recursive (recursively enumerable) if the family has, for all non-recursive degrees $\mathbf{d}$, an enumeration recursive (r.e.) in $\mathbf{d}$. Hence such families $\mathcal{F}$ are fully described by the index set

$$
I_{r}(\mathcal{F}):=\left\{i:(\exists A \in \mathcal{F})\left(\varphi_{i}=\chi_{A}\right)\right\}
$$

or, respectively,

$$
I_{\text {re }}(\mathcal{F}):=\left\{i:(\exists A \in \mathcal{F})\left(W_{i}=A\right)\right\}
$$


Both the index set $I_{r}$ of a family with a recursive enumeration and the index set $I_{r e}$ of a family with a recursively enumerable enumeration are $\Sigma_{3}^{0}$ in the arithmetical hierarchy. Here is the coincidence:

Theorem 2.1. Let $\mathcal{F}$ be a family. If, for every non-recursive degree $\mathbf{d}, \mathcal{F}$ has an enumeration recursive in $\mathbf{d}$, then its index set $I_{r}(\mathcal{F})$ is $\Sigma_{3}^{0}$.

Theorem 2.2 (Jockusch). Let $\mathcal{F}$ be a family. If, for every non-recursive degree $\mathbf{d}$, $\mathcal{F}$ has an enumeration recursively enumerable in $\mathbf{d}$, then its index set $I_{\text {re }}(\mathcal{F})$ is $\Sigma_{3}^{0}$.

Towards giving a sufficient condition under which the implication of her first question holds, Julia Knight defines an extension function for a family $\mathcal{F}$ to be a (possibly partial) function $f: 2^{<\omega} \rightarrow \omega$ such that if $\sigma \in 2^{<\omega}$ and there exists a set $A \in \mathcal{F}$ such that $\chi_{A} \supseteq \sigma$, then $\varphi_{f(\sigma)}=\chi_{A}$ for some such $A$. We mention two facts: Any family with a recursive enumeration has a partial recursive extension function, and so does a family containing all finite sets.

We prove Theorems 2.1 and 2.2 simultaneously with the following two.

Theorem 2.3 (Knight). Let $\mathcal{F}$ be a family which has, for all non-recursive $\mathbf{d}$, an enumeration recursive in $\mathbf{d}$. If $\mathcal{F}$ has a partial recursive extension function, then $\mathcal{F}$ has a recursive enumeration.

Proof (of Theorems 2.3 and 2.1). Let $\mathcal{F}$ be a family which has, for any non-recursive set $X$, an enumeration recursive in $X$. We construct a generic set $D$, attempting to meet the following requirements and expecting to fail. Below, this failure will be exploited to prove the statements of the two theorems for $\mathcal{F}$ separately.

$R_{e}: \varphi_{e}^{D}$ is not the characteristic function of an enumeration of $\mathcal{F}$.

The set $D$ will be Cohen-generic. The set of forcing conditions is $2^{<\omega}$ and the partial order is given by $\subseteq$. We use the old-fashioned notion of a complete forcing sequence (c.f.s.), where $p_{n+1} \supseteq p_{n}$, with $p_{n+1}$ entering the $n$th dense set in some countable collection. $D$ is the set with characteristic function $\bigcup_{n \in \omega} p_{n}$.

Fix a condition $p \in 2^{<\omega}$ and $e \in \omega$. We consider the following four possibilities for $p$ and $e$, showing how extensions of $p$ may force satisfaction of $R_{e}$ in each case.

P1. For some $q \supseteq p, n, x \in \omega, q \vdash \varphi_{e}^{D}(n, x) \downarrow \neq 0,1$, or $q \vdash \varphi_{e}^{D}(n, x) \uparrow$.

We include $q$ in the c.f.s., thereby satisfying $R_{e}$.

P2. For some $q \supseteq p$ and some $n$, for all $q^{\prime} \supseteq q$ there exist $x$ and $r_{0}, r_{1} \supseteq q^{\prime}$ such that $r_{i} \vdash \varphi_{e}^{D}(n, x)=i$.

For each $A \in \mathcal{F}$ the set

$$
D_{A}^{1}:=\left\{r: r \supseteq q \Rightarrow(\exists x)\left(r \vdash \varphi_{e}^{D}(n, x) \neq \chi_{A}(x)\right)\right\}
$$

is dense. We add $q$ to the c.f.s. and enter the sets $D_{A}^{1}$. Then the requirement $R_{e}$ is satisfied.

We write $q \vdash E_{n}=A$ if for all $x$ and all $q^{\prime} \supseteq q$ there is an $r \supseteq q^{\prime}$ such that $r \vdash \varphi_{e}^{D}(n, x) \downarrow=\chi_{A}(x)$. Note that for $q$ and $n$ there is at most one set $A$ such that $q \vdash E_{n}=A$.

P3. For some $q \supseteq p, n \in \omega$ and $B \notin \mathcal{F}$ we have $q \vdash E_{n}=B$.

By putting $q$ into the c.f.s., we meet $R_{e}$.

P4. Not P2 but there exist $A \in \mathcal{F}$ and $q \supseteq p$ such that for all $q^{\prime} \supseteq q$ and all $n$, if $q^{\prime} \vdash E_{n}=B$ then $A \neq B$. 
Since P2 does not hold, the set

$$
D_{n}^{2}:=\left\{q^{\prime}: q^{\prime} \supseteq q \Rightarrow(\exists B)\left(q^{\prime} \vdash E_{n}=B\right)\right\}
$$

is dense for each $n$. By including $q$ in the c.f.s. and entering the sets $D_{n}^{2}$, we meet $R_{e}$.

If for all $p \in 2^{<\omega}$ and $e \in \omega$ one of the cases $\mathrm{P} 1, \ldots, \mathrm{P} 4$ holds, then the forcing construction yields a generic (and so non-recursive) set $D$, in which $\mathcal{F}$ has no recursive enumeration, contrary to the assumption on $\mathcal{F}$.

So let $p \in 2^{<\omega}$ and $e \in \omega$ be such that none of the cases $\mathrm{P} 1, \ldots, \mathrm{P} 4$ hold. It follows that if $q \vdash E_{n}=A$, then $A \in \mathcal{F}$ for all $q \supseteq p, n$ and $A \subseteq \omega$. Moreover, all elements of $\mathcal{F}$ occur in this way.

We first complete the proof of Theorem 2.3. Let $f$ be a partial recursive extension function for $\mathcal{F}$. Fix $n \in \omega$ and $p \subseteq q \in 2^{<\omega}$. Say that $\sigma \in 2^{<\omega}$ has a $q$-computation if there is an $r \supseteq q$ such that $r \vdash \varphi_{e}^{D}(n, x)=\sigma(x)$ for all $x \in \operatorname{dom}(\sigma)$. We make the following observations:

- $q \vdash E_{n}=A$ and $q^{\prime} \supseteq q$ implies $q^{\prime} \vdash E_{n}=A$.

- The set $\left\{\sigma \in 2^{<\omega}: \sigma\right.$ has a $q$-computation $\}$ is r.e. (uniformly in $q$ ).

- By definition, $q \vdash E_{n}=A$ if and only if any $\sigma \in 2^{<\omega}$ has a $q$-computation if and only if $\sigma \subseteq \chi_{A}$.

- Since $p, e$ do not satisfy $\mathrm{P} 1$, for any $q^{\prime} \supseteq q$ there is a $\sigma \in 2^{<\omega}$ which has a $q^{\prime}$-computation.

- The previous two items imply that the predicate $q \supseteq p \Rightarrow(\forall A)\left(q \forall E_{n}=A\right)$ in $q$ and $n$ is r.e.

- Since $p, e$ do not satisfy P3, if $q \supseteq p$ and $q \nvdash E_{n}=A$ for any $A \in \mathcal{F}$, then for any $\sigma \in 2^{<\omega}$ with a $q$-computation there is a set $A \in \mathcal{F}$ such that $\sigma \subseteq \chi_{A}$.

We form a recursive enumeration $R$ of $\mathcal{F}$, using pairs $(q, n)$ as indices, where $q \in 2^{<\omega}$ with $q \supseteq p$ and $n \in \omega$. Since there is a recursive bijection between $P \times \omega$ and $\omega$, where $P=\left\{q \in 2^{<\omega}: q \supseteq p\right\}$, this suffices. Let $\left(\sigma_{q}^{(n)}\right)_{n \in \omega}$ be an effective enumeration of $\left\{\sigma \in 2^{<\omega}: \sigma\right.$ has a $q$-computation $\}$ by the second observation. We choose this enumeration so that, additionally, for every $\sigma$ with a $q$-computation there are infinitely many $i$ such that $\sigma_{q}^{(i)}=\sigma$. Define a partial recursive function $a_{q}$ by $a_{q}(0):=0$ and $a_{q}(n+1):=\mu m>n . \sigma_{q}^{(m)} \supseteq \sigma_{q}^{\left(a_{q}(n)\right)}$. By the fourth observation, let $h: \omega \rightarrow 2^{<\omega} \times \omega$ be a recursive function with range $\left\{(q, n): q \supseteq p\right.$ and $\left.(\forall A)\left(q \forall E_{n}=A\right)\right\}$.

$R_{(q, n)}$ is defined by

$R_{(q, n)}:= \begin{cases}\bigcup_{0 \leq i \leq m} \sigma_{q}^{\left(a_{q}(i)\right)} \cup \varphi_{f\left(\sigma_{q}^{a_{q}(m)}\right)} & \text { if } m \text { is least such that } h(m)=(q, n), \\ \bigcup_{i \in \omega} \sigma_{q}^{\left(a_{q}(i)\right)} & \text { otherwise. }\end{cases}$

Fix $q \supseteq p$ and $n \in \omega$. By definition of the functions $h$, there is $m$ satisfying the first case if and only if $(\forall A)\left(q \forall E_{n}=A\right)$. If $q \vdash E_{n}=A$, then the choice of the enumeration $\left(\sigma_{q}^{(n)}\right)_{q \in 2<\omega, n \in \omega}$ and the function a guarantees $R_{(q, n)}=A$. If $q \forall E_{n}=A$ for any $A$, then by the fact that $f$ is an extension function for $\mathcal{F}$, it follows that $R_{(q, n)} \in \mathcal{F}$.

Since for every $A \in \mathcal{F}$ there are $q$ and $n$ such that $q \vdash E_{n}=A$, it follows that $R$ is an enumeration of $\mathcal{F}$, and Theorem 2.3 is proved. 
We turn to the proof of Theorem 2.1. As mentioned above, if none of the cases holds for $p$ and $e$, then we have

$$
\mathcal{F}=\left\{X:(\exists q \supseteq p)(\exists n)\left(q \vdash E_{n}=X\right)\right\} .
$$

The ternary relation $\left(q \vdash E_{n}=X\right) \wedge\left(\chi_{X}=\varphi_{i}\right)$ (in $q, n$ and $i$ ), when restricted to $i$ such that $\varphi_{i}$ is the characteristic function of a set, is $\Pi_{2}^{0}$ so that $I_{r}(\mathcal{F})$ is $\Sigma_{3}^{0}$.

Theorem 2.4 (Knight). Let $\mathcal{F}$ be a family such that for all non-recursive $X, \mathcal{F}$ has an enumeration r.e. in $X$. If $\mathcal{F}$ contains all finite sets, then $\mathcal{F}$ has an r.e. enumeration.

Proof of Theorems 2.4 and 2.2. As above, we construct a generic set $D$, attempting to meet the following requirements and expecting to fail.

$$
R_{e} . W_{e}^{D} \text { is not an enumeration of } \mathcal{F} \text {. }
$$

We use the same forcing notion as was used for the previous proof. Fix $p \in 2^{<\omega}$ and $e \in \omega$. We consider the following three cases.

C1. For some $q \supseteq p$ and some $n \in \omega$, for all $q^{\prime} \supseteq q$, there exist $x \in \omega$ and $r_{0}, r_{1} \supseteq q^{\prime}$ such that $r_{0} \vdash(n, x) \in W_{e}^{D}$ and $r_{1} \vdash(n, x) \notin W_{e}^{D}$.

For each $A \in \mathcal{F}$ the set

$$
\begin{aligned}
D_{A}^{1}:=\{r: r \supseteq q \Rightarrow(\exists x)[(r \vdash(n, x) & \left.\in W_{e}^{D} \wedge x \notin A\right) \text { or } \\
(r \vdash(n, x) & \left.\left.\left.\notin W_{e}^{D} \wedge x \in A\right)\right]\right\}
\end{aligned}
$$

is dense. We put $q$ into the c.f.s. and enter the sets $D_{A}^{1}$. Requirement $R_{e}$ is satisfied.

We write $q \vdash E_{n}=A$ if for all $x$, if $x \in A$, then for all $q^{\prime} \supseteq q$ there is $r \supseteq q^{\prime}$ such that $r \vdash(n, x) \in W_{e}^{D}$, and if $x \notin A$ then $q \vdash(n, x) \notin W_{e}^{D}$.

C2. Case $\mathrm{C} 1$ does not hold, but $q \vdash E_{n}=B$ for some $q \supseteq p, n \in \omega$ and $B \notin \mathcal{F}$. Requirement $R_{e}$ is met by putting $q$ in the c.f.s.

C3. Case $\mathrm{C} 1$ does not hold, but there exists $A \in \mathcal{F}$ such that for all $q^{\prime} \supseteq q$ and all $n$, if $q^{\prime} \vdash E_{n}=B$, then $A \neq B$.

Since Case C1 does not hold, the set

$$
D_{n}^{2}:=\left\{q^{\prime}: q^{\prime} \supseteq q \Rightarrow(\exists B)\left(q^{\prime} \vdash E_{n}=B\right)\right\}
$$

is dense for all $n$. We meet the requirement $R_{n}$ by including $q$ in the c.f.s. and entering all sets $D_{n}^{2}$.

If for all $p \in 2^{<\omega}$ and $e \in \omega$ one of the cases C1,C2, or C3 holds, then the forcing construction yields a generic (and hence non-recursive) set $D$, in which $\mathcal{F}$ has no r.e. enumeration. This contradicts the assumption of both Theorem 2.2 and Theorem 2.4. So let $p \in 2^{<\omega}$ and $e \in \omega$ be such that none of the cases C1,C2,C3 holds. We show that the index set $I_{r e}$ of $\mathcal{F}$ is $\Sigma_{3}^{0}$. As in the proof of Theorem 2.3, for all $q \supseteq p, n$, and $A \subseteq \omega$, if $q \vdash E_{n}=A$, then $A \in \mathcal{F}$, and all members of $\mathcal{F}$ occur in this way. Therefore we have

$$
\left\{i: W_{i} \in \mathcal{F}\right\}=\left\{i:(\exists q \supseteq p)(\exists n)\left(q \vdash E_{n}=W_{i}\right)\right\} .
$$

The relation " $q \vdash E_{n}=W_{i}$ " is $\Pi_{2}^{0}$, and so the index set of $\mathcal{F}$ is $\Sigma_{3}^{0}$. This completes the proof of Theorem 2.2. To complete the proof of Theorem 2.4, note that since $\mathcal{F}$ includes all finite sets and only contains r.e. sets, by a theorem of Yates $[7$, Theorem 8], $\mathcal{F}$ has an r.e. enumeration. 


\section{A FAMILY OF FINITE SETS}

In this section we first define a family $\mathcal{C}$ which has no r.e. enumeration. Then we show that for every non-recursive set $X$ there is an enumeration of $\mathcal{C}$ which is recursive in $X$, and finally that every non-recursive degree contains an enumeration of $\mathcal{C}$. This corrects an earlier statement in [6, p 187]. In particular, we apologize for connecting the error with Martin Kummer.

Let $r$ be the partial recursive function defined by

$$
r(e):=\left(\mu\langle i, x, s\rangle \cdot\langle e, x\rangle \in \Omega_{i, s}^{(e)}\right)_{.1} .
$$

Informally, the value of $r(e)$ is the first index $i$ to be found such that there is a number of the form $\langle e, x\rangle \in \Omega_{i}^{(e)}$; if there is no such $i$ then $r(e)$ is not defined. Let the family $\mathcal{C}$ be defined by

$$
\mathcal{C}:=\{\langle e, A\rangle: A \text { is finite, } e \in \omega\}-\left\{\langle e, \omega\rangle \cap \Omega_{r(e)}^{(e)}: r(e) \downarrow\right\} .
$$

$\mathcal{C}$ does not have an r.e. enumeration; for suppose $\Omega^{\left(e_{0}\right)}$ is an enumeration of $\mathcal{C}$. Then $r\left(e_{0}\right)$ is defined, and the set $\Omega_{r\left(e_{0}\right)}^{\left(e_{0}\right)}=\langle e, \omega\rangle \cap \Omega_{r\left(e_{0}\right)}^{\left(e_{0}\right)}$ is not a member of $\mathcal{C}$.

Let $X$ be an arbitrary non-recursive set. To see how to construct an enumeration $S^{X}$ of $\mathcal{C}$ such that $S^{X} \leq_{T} X$ we use the following lemma.

Lemma 3.1. Uniformly in $i$ and recursively in $X$ there is a finite set $A_{i}^{X} \leq_{T} X$ such that $W_{i} \neq A_{i}^{X}$.

Let $g$ be a partial recursive function such that $g(e) \downarrow$ if and only if $r(e) \downarrow$ and if $r(e) \downarrow$ then $W_{g(e)}=\left(\langle e, \omega\rangle \cap \Omega_{r(e)}^{(e)}\right) .2$. Let $h$ be a partial recursive function such that $h(e, a) \downarrow$ if and only if $g(e) \downarrow$ and if $g(e) \downarrow$ then $W_{h(e, a)}=W_{g(e)}-a$.

Define

$$
S_{\langle n, t, e\rangle}^{X}:=\left\langle e, B^{X}(n, t, e)\right\rangle,
$$

where

$$
B^{X}(n, t, e):= \begin{cases}\left.D_{n} \cup\left(s_{0}+A_{h\left(e, s_{0}\right)}^{X}\right)\right) & \text { if there is } s>t, \max \left(D_{n}\right)+1 \\ & \text { such that } g_{s}(e) \downarrow \text { and } W_{g(e), s}=D_{n}, \\ & \text { and } s_{0} \text { is the least such, } \\ & \text { otherwise. }\end{cases}
$$

By the lemma, $S^{X}$ is recursive in $X$. We claim that $S^{X}$ is an enumeration of $\mathcal{C}$.

"ᄃ". First of all, it follows from the lemma that all sets $B^{X}(n, t, e)$ are finite and so the family enumerated by $S^{X}$ is contained in $\{\langle e, A\rangle: A$ is finite $\}$.

Suppose $r(e) \downarrow$ and $S_{\langle n, t, e\rangle}^{X}=\langle e, \omega\rangle \cap \Omega_{r(e)}^{(e)}$. It follows that $B^{X}(n, t, e)=W_{g(e)}$. There has to be a stage $s>t, \max \left(D_{n}\right)+1$ such that $W_{g(e), s}=D_{n}$, because otherwise $B^{X}(n, t, e)=D_{n} \neq W_{g(e)}$, a contradiction. Let $s_{0}$ be the least such $s$. Then $B^{X}(n, t, e)=D_{n} \cup\left(s_{0}+A_{h\left(e, s_{0}\right)}^{X}\right)=W_{g(e)}$, so that $A_{h\left(e, s_{0}\right)}^{X}=W_{h\left(e, s_{0}\right)}$, a contradiction.

"卫". Let $e, n \in \omega$ such that $C=\left\langle e, D_{n}\right\rangle \in \mathcal{C}$. We want to find a number $z$ such that $S_{z}^{X}=C$. Let $t$ be such that $W_{g(e), s} \neq D_{n}$ for all $s>t$. (If there is no such $t$ then $C \notin \mathcal{C}$.) By definition, $B^{X}(n, t, e)=D_{n}$, so that $S_{\langle n, t, e\rangle}^{X}=C$.

We have constructed an enumeration of $\mathcal{C}$ which is recursive in $X$. Simple coding is sufficient to obtain an enumeration T-equivalent to $X$ : Choose $A, B \in \mathcal{C}$ so that 
$A-B \neq \emptyset$. Define another enumeration of $\mathcal{C}$,

$$
P_{2 n}^{X}:=S_{n}^{X}, \quad P_{2 n+1}^{X}:= \begin{cases}A & \text { if } n \in X, \\ B & \text { if } n \notin X .\end{cases}
$$

Clearly, $P^{X}$ is recursive in $X$ and enumerates $\mathcal{C}$. Let $x \in A-B$. Then $z \in X$ if and only $x \in P_{2 z+1}^{X}$, so that $X$ is recursive in $P^{X}$.

Proof of Lemma 3.1. Let $y, n: \omega^{2} \rightarrow \omega$ be two recursive one-one functions such that their ranges are disjoint and cover $\omega$. Let

$$
\alpha(s, x):= \begin{cases}y(s, x) & \text { if } x \in X, \\ n(s, x) & \text { otherwise, }\end{cases}
$$

so that $\alpha \leq_{T} X$. We construct the set $A_{i}^{X}$ in stages uniformly in $i$ and recursively in $X$. In the course of the construction, numbers $a_{x}$ may become defined.

Stage 0. Set $x:=0 . A_{i, 0}^{X}$ is empty.

Stage $s+1$. If $W_{i, s} \neq A_{i, s}^{X}$, pass to the next stage. Otherwise enumerate $\alpha(s, x)$ in $A_{i}^{X}$, let $a_{x}:=s$ and increase $x$ by one.

End of construction.

The set $A_{i}^{X}$ is (by construction) r.e. in $X$. It is also recursive in $X$ : By inspection, $A_{i}^{X}$ only contains numbers $\alpha(a, b)$. If $y(s, x) \in A_{i}^{X}$, then $y(s, x) \in A_{i, s+1}^{X}$ and the same holds for numbers $n(s, x)$. This together with the choice of $y$ and $n$ is sufficient.

Claim 1. The set $A_{i}^{X}$ is finite.

Suppose $A_{i}^{X}$ is infinite. During the construction of $A_{i}^{X}$ infinitely many numbers $a_{x}$ are defined. By induction on $x$, it follows that $A_{i, a_{x}}^{X}=\left\{\alpha\left(a_{j}, j\right): j \leq x\right\}$, and therefore $W_{i, a_{x}}=\left\{\alpha\left(a_{j}, j\right): j<x\right\}$ for all $x \in \omega$. Thus, $W_{i}=\left\{\alpha\left(a_{x}, x\right): x \in \omega\right\}$. Now, $x \in X$ if and only if $(\exists t)\left(y(t, x) \in W_{i}\right)$, and $x \notin X$ if and only if $(\exists t)(n(t, x) \in$ $\left.W_{i}\right)$. This means that $X$ is recursive, a contradiction.

Claim 2. $A_{i}^{X}$ is different from $W_{i}$.

By the previous claim it is sufficient to consider the case when $W_{i}$ is finite. Let $s_{0}$ be the least number such that $W_{i, s_{0}}=W_{i}$. Then either $W_{i, s_{0}} \neq A_{i, s_{0}}^{X}$, in which case $W_{i, s_{0}} \neq A_{i, s_{0}}^{X}=A_{i}^{X}$; or $W_{i, s_{0}}=A_{i, s_{0}}^{X}$, so that at stage $s_{1}=s_{0}+1$ a new number is enumerated in $A_{i}^{X}$, whence $W_{i}=W_{i, s_{1}}=A_{i, s_{0}}^{X} \neq A_{i}^{X}$.

Lemma 3.1 is proved.

\section{Applichtion to Lempp's Question}

Let $\mathcal{F}$ be a family. With $\mathcal{F}$ we associate the following countable structure $\mathfrak{A}_{\mathcal{F}}$ in the language $L=(S, Z, I)$, where $S$ is a binary predicate symbol and $Z$ and $I$ are unary predicate symbols. The universe of $\mathfrak{A}_{\mathcal{F}}$ is $\mathcal{F} \times \omega \times \omega$. For every $A \in \mathcal{F}$, set $Z((A, x, 0))$ and $S((A, x, n),(A, x, n+1))$. Set $I((A, x, n))$ if and only if $n \in A$. Thus, countably many $S$-chains $(A, x, 0),(A, x, 1),(A, x, 2), \ldots$ are associated with every $A \in \mathcal{F}$, and in every chain $I$ holds of the $n$-th member if $n \in A$.

Theorem 4.1. Let $\mathbf{d}$ be a Turing degree and $\mathcal{F}$ be a family. Then $\mathcal{F}$ has an enumeration recursive in $\mathbf{d}$ if and only if the structure $\mathfrak{A}_{\mathcal{F}}$ has a representation recursive in $\mathbf{d}$. 
Proof. " $\Rightarrow$ ". Let $Q^{X}$ be an enumeration of $\mathcal{F}$ which is recursive in $X \in \mathbf{d}$. Define $R^{X}$ to be another $X$-recursive enumeration of $\mathcal{F}$ by $R_{\langle n, i\rangle}^{X}:=Q_{i}^{X}$. A representation of $\mathfrak{A}_{\mathcal{F}}$ is given by $\mathfrak{B}$, where $Z_{\mathfrak{B}}(\langle x, 0\rangle), S_{\mathfrak{B}}(\langle x, n\rangle,\langle x, n+1\rangle)$, and $I_{\mathfrak{B}}(\langle x, n\rangle)$ if and only if $x \in R_{n}^{X}$. These predicates are recursive in $R^{X}$, and therefore $D(\mathfrak{B})$ is recursive in $X$.

" $\Leftarrow$ ". Let $\mathfrak{B}$ be a representation of $\mathfrak{A}_{\mathcal{F}}$ whose open diagram is recursive in $X \in \mathbf{d}$. The set $Z:=\left\{x: Z_{\mathfrak{B}}(x)\right\}$ is recursive in $X$. With each $x \in Z$ we associate the set $S_{x}:=\left\{n: I_{\mathfrak{B}}\left(f^{(n)}(x)\right)\right\}$, where $f(x)$ is the unique $y$ such that $S_{\mathfrak{B}}(x, y)$, and $f^{(n)}(x)$ denotes the $n$-fold application of $f$ to $x$. The sets $S_{x}$ are uniformly in $x \in Z$ recursive in $X$, and, by definition of $\mathfrak{A}_{\mathcal{F}}, \mathcal{F}=\left\{S_{x}: x \in Z\right\}$. This suffices.

Corollary 4.2 (Slaman [4]). There is a structure which has representations only in the non-recursive degrees.

Proof. Apply the theorem to the family $\mathcal{C}$ defined in the previous section to obtain representations of $\mathfrak{A}_{\mathcal{C}}$ below any non-recursive T-degree. Apply Theorem 1.1 to obtain representations in all non-recursive T-degrees.

Slaman remarked (private communication) that the construction given in [4] yields a structure which is not elementarily equivalent to any recursively represented structure. Informally, the reason for this is as follows. Essentially, the construction proceeds in such a way that the final outcome of the actions taken to diagonalize against the recursive representations can be read off the theory of the structure (which is called $\mathfrak{M}$ ):

He writes ([4, Section 2.1]): "We will ensure that either $R^{-1}\left(T_{i}\right)^{\Re_{i}}=\emptyset$, or $\left\langle T_{i},<_{L}\left\lceil T_{i}\right\rangle^{\mathfrak{R}_{i}}\right.$ is not isomorphic to $\left\langle T_{i},<_{L}\left\lceil T_{i}\right\rangle^{\mathfrak{M}}\right.$, or there is a $p$ in $R^{-1}\left(T_{i}\right)^{\mathfrak{R}_{i}}$ such that $\zeta(p)^{\mathfrak{R}_{i}}$ is not maximal, or there is a $p$ in $R^{-1}\left(T_{i}\right)^{\mathfrak{R}_{i}}$ such that $\zeta(p)^{\mathfrak{R}_{i}}$ is infinite. Since none of these disjuncts apply to $\mathfrak{M}$, we will thus ensure that $\mathfrak{M}$ has no recursive presentation."

Fix $i \in \omega$. The first and third disjunct can be directly formalized in the language $\mathfrak{L}$ of the structure. If the second disjunct holds, then the construction $[4$, Section 2.2.1] yields a tree $\left\langle T_{i},<_{L}\left\lceil T_{i}\right\rangle^{\mathfrak{M}}\right.$ which is finite. Therefore this tree is described by a sentence in the theory of $\mathfrak{M}$. The fourth disjunct also cannot be formalized in $\mathfrak{L}$, but, provided the first three disjuncts are not true, the strategy used in [4, Section 2.2.2] results in

$$
(\exists p)\left(R\left(p, s^{i}(0)\right) \wedge(\forall x)\left(R(p, x) \rightarrow(\exists y)\left(x<_{T} y \wedge R(p, y)\right)\right)\right)
$$

being true in $\mathfrak{R}_{i}$. This is a formula in $\mathfrak{L}$, and (by the same strategy) not true in $\mathfrak{M}$. Hence there is no recursively represented structure which is elementarily equivalent to $\mathfrak{M}$.

The structure obtained from the family $\mathcal{C}$ by Theorem 4.1 is of a different kind:

Theorem 4.3. There is a structure which has representations only in the nonrecursive degrees and has a recursively represented elementary extension.

Proof. Let us look at the following family:

$$
\mathcal{D}:=\{\langle e, A\rangle: A \text { is finite, } e \in \omega\} .
$$

Obviously, $\mathcal{D}$ has a recursive enumeration, and $\mathfrak{A}_{\mathcal{D}}$ has a recursive representation. At the same time, the structure $\mathfrak{A}_{\mathcal{C}}$ from the proof of Corollary 4.2 is contained in $\mathfrak{A}_{\mathcal{D}}$, and they are elementarily equivalent: 
It suffices to show that for any formula $(\exists x)(\phi)$ in variables $x_{1}, \ldots, x_{n}$, if

$$
\mathfrak{A}_{\mathcal{D}} \models(\exists x)(\phi)\left[x_{1}:=c_{1}, \ldots, x_{n}:=c_{n}\right]
$$

and $c_{1}, \ldots c_{n} \in \mathfrak{A}_{\mathcal{C}}$, then there is $c \in \mathfrak{A}_{\mathcal{C}}$ such that

$$
\mathfrak{A}_{\mathcal{D}}=\phi\left[x:=c, x_{1}:=c_{1}, \ldots, x_{n}:=c_{n}\right] .
$$

Note that if $\mathfrak{A}_{\mathcal{D}}=(\exists x)(\phi)\left[x_{1}:=c_{1}, \ldots, x_{n}:=c_{n}\right]$, then there is a $d$ in $\mathfrak{A}_{\mathcal{D}}$ such that either

$$
\begin{aligned}
\mathfrak{A}_{\mathcal{D}} \models & \phi\left[x:=d, x_{1}:=c_{1}, \ldots, x_{n}:=c_{n}\right] \\
\wedge & \wedge S\left(d, c_{1}\right) \wedge \neg S\left(c_{1}, d\right) \wedge \ldots \wedge \neg S\left(d, c_{n}\right) \wedge \neg S\left(c_{n}, d\right),
\end{aligned}
$$

or

$$
\begin{aligned}
\mathfrak{A}_{\mathcal{D}} \models & \phi\left[x:=d, x_{1}:=c_{1}, \ldots, x_{n}:=c_{n}\right] \\
& \wedge\left(S\left(d, c_{1}\right) \vee S\left(c_{1}, d\right) \vee \ldots \vee S\left(d, c_{n}\right) \vee S\left(c_{n}, d\right)\right) .
\end{aligned}
$$

In the former case, choose $c$ from $\mathfrak{A}_{\mathcal{C}}$ outside of the $S$-chains of $\mathfrak{A}_{\mathcal{C}}$ which $c_{1}, \ldots, c_{n}$ belong to such that it satisfies $Z$ and $I$ in the same way $d$ does. In the latter case, $d$ is already part of an $S$-chain which is contained in $\mathfrak{A}_{\mathcal{C}}$, and so an element of $\mathfrak{A}_{\mathcal{C}}$.

\section{REFERENCES}

[1] Christopher J. Ash, Carl G. Jockusch, jr. and Julia F. Knight; Jumps of orderings, Trans. Amer. Math. Soc., vol. 319, (1990), p. 573 - 599. MR 90j:03081

[2] Julia F. Knight; Degrees Coded in Jumps of Orderings, J. Symbolic Logic, vol. 51, (1986), p. 1034 - 1042. MR 88j:03030

[3] Linda J. Richter; Degrees of Structures, J. Symbolic Logic, vol. 46 (1981), p. 723 - 731. MR 83d:03048

[4] Theodore A. Slaman; Relative to any Nonrecursive Set, Proc. Amer. Math. Soc., vol. 126 (1998), 2117-2122. CMP 97:11

[5] Robert I. Soare; Recursively Enumerable Sets and Degrees, Springer Verlag, Berlin, Heidelberg, New York, Tokyo, 1987. MR 88m:03003

[6] Stephan Wehner; On Injective Enumerability of Recursively Enumerable Classes of Cofinite Sets, Arch. Math. Logic, vol. 34, (1995), p. 183 - 196. MR 96d:03062

[7] C.E.M. Yates; On the Degrees of Index Sets II, Trans. Amer. Math. Soc., vol. 135 (1969), p. 249 - 266. MR 39:2637

Department of Chemistry, University of British Columbia, Vancouver, British Columbia, Canada V6T 1Z1

E-mail address: stephan@pepe.chem.ubc.ca 